\title{
Solving Fuzzy Games Problems by Using Ranking Functions
}

\author{
Iden Hasan Hussein ${ }^{1} \quad$ Zainab saad Abood ${ }^{2 *}$
}

Received 24/4/2017

Accepted 18/9/2017

This work is licensed under a Creative Commons Attribution 4.0 International License.

\begin{abstract}
:
In this paper, we deal with games of fuzzy payoffs problem while there is uncertainty in data. We use the trapezoidal membership function to transform the data into fuzzy numbers and utilize the three different ranking function algorithms. Then we compare between these three ranking algorithms by using trapezoidal fuzzy numbers for the decision maker to get the best gains.
\end{abstract}

Keywords: Fuzzy numbers, Fuzzy games problem, Trapezoidal membership function, three ranking functions.

\section{Introduction:}

The theory of games is a mathematical theory that deals with general features of competitive situations, (1). It is usually used when two or more individuals or organizations with conflicting objectives try to make decisions; it's based on the minimax-principle, (2). The set of objective functions in the game, that may have uncertain values where the way to deal with uncertainty, is to use the concept of fuzzy games, (3). A ranking function is used which helps us not just to find the solution, but also to find the best gain for the fully fuzzy game problem.

Many authors studied fuzzy games in which some of them with ranking functions to solve the games problem. Aristidou. M, Sarangi. S at (2005) represented a non-cooperative model of a normal form game using tools from fuzzy set theory, (4). Gao. J at (2007) represented a strategic game with fuzzy payoffs, (5). Medinechiene. M, Zavadskas. E. K, Turskis. Z at (2011) described a model of dwelling selection using fuzzy games theory on buildings, (6). Jawad. M. A at (2012) represented fuzzy sets and fuzzy processes with game theory to address the uncertainty in data for mobile phone companies in Iraq, (3). Kumar. R. S, Kumaraghura. $S$ at (2015) represented a solution of fuzzy game problem with triangular fuzzy numbers using a ranking function to compare the fuzzy numbers, (7). Selvakumari. K, Lavanya. S at (2015) considered a two zero sum game with imprecise (triangular or trapezoidal) fuzzy numbers using ranking function for an approach to solve the problem, (8).

${ }^{1,2}$ Department of Mathematics, College of Science for Women University of Baghdad, Iraq.

*Corresponding authors: zainb.saad81@gmail.com
Kumar. R. S, Gnanaprakash. K at (2016) represented a two zero sum game of order $(3 \times 3)$ with octagonal fuzzy payoffs using ranking function to solve the fuzzy game, (2).

The objective of this paper is to compare three different ranking algorithms to select the best (highest) gains for the fuzzy game problem using trapezoidal fuzzy numbers. This paper contains five sections: in section two a review of some fuzzy theory concepts is given. Section three defines the fuzzy games problem and its formula. Section four presents the ranking function and some properties and three algorithms of ranking function. Finally in section five a numerical example is represented depending on the three different ranking functions.

\section{Basic Concept of Fuzzy Set Theory:}

In this section we will introduce some definitions of fuzzy set theory.

Definition, (2):

Let $\mathrm{X}$ be a nonempty set, a fuzzy set $\tilde{A}$ in $\mathrm{X}$ is characterized by its membership function $\mathrm{A}=$ $\rightarrow[0,1]$ and $\mathrm{A}(x)$ is interpreted as the degree of membership of element $\mathrm{X}$ in fuzzy $\tilde{A}$ for each $x \in$ $X$.

\section{Definition, (9):}

The crisp set of elements that belong to the fuzzy set $\tilde{A}$ at least to the degree $\alpha$ is called the $\alpha$-level set

$$
A_{\alpha}=\left\{x \in X / \mu_{\tilde{A}} \geq \alpha\right\}
$$

$\hat{A}_{\alpha}=\left\{x \in X / \mu_{\tilde{A}}>\alpha\right.$ \}is called strong $\alpha$-level set

Definition,(9): or strong $\alpha$-cut.

A fuzzy set $\tilde{A}$ is convex in $\mathrm{R}$ if $\mu_{\tilde{A}}\left(\alpha x_{1}+(1-\alpha) x_{2}\right) \geq \min \left\{\mu_{\tilde{A}}\left(x_{1}\right), \mu_{\tilde{A}}\left(x_{2}\right)\right\}$ 
For all $x_{1}, x_{2} \in X, \alpha \in[0,1]$.

Definition, (10):

A fuzzy set is called normal if hgt $(\tilde{A})=1$. A nonempty fuzzy set can always be normalized by dividing $\mathrm{A}(x)$ by hgt (A).

\section{Definition,(8):}

A fuzzy number $\tilde{A}$ is a fuzzy set on the real line $R$, must satisfy the following conditions:

1 - There exists at least one $x_{0} \in R$ with $\mu_{\tilde{A}}\left(x_{0}\right)=1$.

2- $\mu_{\tilde{A}}(x)$ is piecewise continuous.

3- Âmust be normal and convex.

\section{Definition, (11):}

A fuzzy number $\tilde{A}=(a, b, c, d ; 1)$, where a, b, $\mathrm{c}, \mathrm{d}$ are real numbers, is said to be a trapezoidal fuzzy number if its membership function is given by:

$$
\mu_{\tilde{A}_{(x)}}=\left\{\begin{array}{lc}
\frac{x-a}{b-a} & , \quad a \leq x \leq b \\
1 & , \quad b \leq x \leq c \\
\frac{d-x}{d-c} & , \quad c \leq x \leq d \\
0 & , \quad \text { otherwise }
\end{array}\right.
$$

\section{Fuzzy Game Problem}

The fuzzy game problem is where all the payoffs $\left(\tilde{a}_{i j}\right)$ of the game matrix are trapezoidal fuzzy quantities. Now the formula of the fully fuzzy game problem is:

\section{Player B}

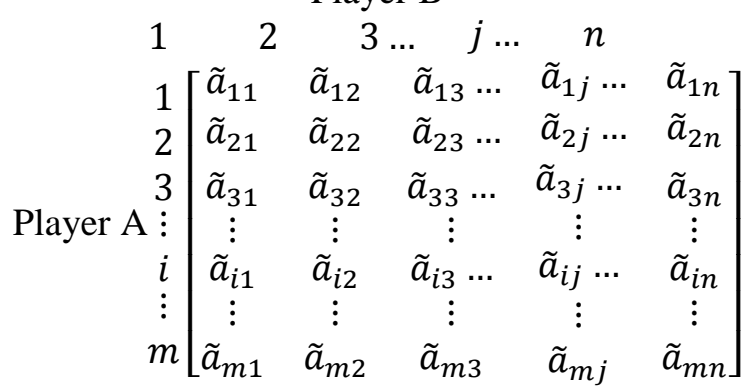

\section{Ranking Function (12)}

If $F(R)$ is the set of all fuzzy numbers defined on the set of real numbers $R$, then a ranking function $R: F(R) \rightarrow R$ maps each fuzzy number into a real ordinary number where there is a natural order, the order rules are:

- $\quad \tilde{A}>\tilde{B}$ if and only if $\mathrm{R}(\tilde{A})>\mathrm{R}(\tilde{B})$.

- $\tilde{A}=\tilde{B}$ if and only if $\mathrm{R}(\tilde{A})=\mathrm{R}(\tilde{B})$.

- $\tilde{A}<\tilde{B}$ if and only if $\mathrm{R}(\tilde{A})<\mathrm{R}(\tilde{B})$.

Where $\tilde{A}$ and $\tilde{B}$ are two fuzzy numbers belong to $\mathrm{F}(\mathrm{R})$.

\section{Algorithms of Ranking Function}

- The First Algorithm (13, 14):

We use the previous trapezoidal membership function and by using the $\alpha$-cut where $\alpha \in[0,1]$ then

$$
\begin{aligned}
& \alpha=\frac{x-a}{b-a} \Rightarrow x=\alpha(b-a)+a=\inf \tilde{A}_{(\alpha)} . \\
& \alpha=\frac{d-x}{d-c} \Rightarrow x=d-\alpha(d-c)=\sup \tilde{A}_{(\alpha)} .
\end{aligned}
$$

Applying the ranking function $\mathrm{R}(\tilde{A})=$ $\frac{1}{2} \int_{0}^{1}\left(\inf \tilde{A}_{(\alpha)}+\sup \tilde{A}_{(\alpha)}\right) d_{\alpha}, \alpha \in[0,1]$ then $\mathrm{R}(\tilde{A})$ becomes the following equation:

$$
\begin{aligned}
& \mathrm{R}(\tilde{A})=\frac{1}{2} \int_{0}^{1}[\alpha(b-a)+a+d-\alpha(d-c)] d_{\alpha} \\
& \mathrm{R}(\tilde{A})=\frac{1}{2}\left[\frac{\alpha^{2}}{2}(b-a)+\alpha a+\alpha d-\frac{\alpha^{2}}{2}(d-c)\right]_{0}^{1} \\
& \mathrm{R}(\tilde{A})=\frac{1}{2}\left[\frac{1}{2} b-\frac{1}{2} a+a+d-\frac{1}{2} d+\frac{1}{2} c\right] \\
& \mathrm{R}(\tilde{A})=\frac{a+b+c+d}{4}
\end{aligned}
$$

\section{- The Second Algorithm (13,15):}

The following membership is used:

$$
\mu_{\tilde{A}_{(x)}}=\left\{\begin{array}{cl}
1-\frac{a-x}{c} & , \quad a-c \leq x \leq a \\
1 & , \quad a \leq x \leq b \\
1-\frac{x-b}{d} & , \quad b \leq x \leq b+d \\
0 & , \quad \text { otherwise }
\end{array}\right.
$$

By using the $\alpha$-cut where $\alpha \in[0,1]$ then

$$
\begin{aligned}
& 1-\frac{a-x}{c}=\alpha \Rightarrow x=a+\alpha c-c=\inf \tilde{A}_{(\alpha)} \\
& 1-\frac{x-b}{d}=\alpha \Rightarrow x=d-\alpha d+b=\sup \tilde{A}_{(\alpha)}
\end{aligned}
$$

Applying the ranking function and when $c \neq d$ then the ranking function is:

$\mathrm{R}(\tilde{A})=\frac{2 a+2 b+d-c}{4}$

\section{- The Third Algorithm(16):}

the following membership function is used:

$$
\mu_{\tilde{A}_{(x)}}=\left\{\begin{array}{cl}
\frac{w(x-a)}{(b-a)}, & a \leq x \leq b \\
1, & \mathrm{~b} \leq x \leq c \\
\frac{w(d-x)}{(d-c)}, & c \leq x \leq d \\
0, & \text { otherwise }
\end{array}\right.
$$

By using the $\alpha$-cut where $\alpha \in[0,1]$ then

$$
\begin{gathered}
\frac{w(x-a)}{(b-a)}=\alpha \Rightarrow x=\frac{\alpha(b-a)}{w}=\inf \tilde{A}_{(\alpha)} \\
\frac{w(d-x)}{(d-c)}=\alpha \Rightarrow x=d-\frac{\alpha(b-c)}{w}=\sup \tilde{A}_{(\alpha)}
\end{gathered}
$$

Applying the following ranking function $\mathrm{R}(\tilde{A})=$ $\frac{1}{2} \int_{0}^{w}\left[\inf \tilde{A}_{(\alpha)}+\sup \tilde{A}_{(\alpha)}\right] d_{\alpha}$ then

$$
\begin{aligned}
& \mathrm{R}(\tilde{A})=\frac{1}{2} \int_{0}^{w}\left[\frac{\alpha(b-a)}{w}+a+d-\frac{\alpha(d-c)}{w}\right] d_{\alpha} \\
& \mathrm{R}(\tilde{A})=\frac{1}{2}\left[\frac{\alpha^{2}(b-a)}{2 w}+a \alpha+d \alpha-\frac{\alpha^{2}(d-c)}{2 w}\right]_{0}^{w}
\end{aligned}
$$


$\mathrm{R}(\tilde{A})=\frac{1}{2}\left[\frac{w^{2}(b-a)}{w}+2 w a+2 w d-\frac{w^{2}(d-c)}{w}\right]$
$\mathrm{R}(\tilde{A})=\frac{w}{4}(a+b+c+d)$.

Numerical Example (17)

This example may clarify the ranking algorithms.

Solve the game whose payoff matrix is: Player B

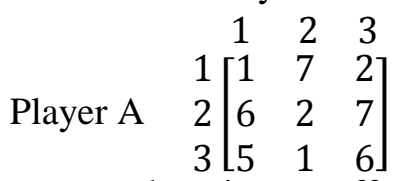

Which represents the crisp payoff game matrix.

Case1: The crisp game matrix is solved in page (505) (17), where the value of the game is $v=4$ units of money with an optimal strategy for player A $(0.4,0.6,0)$ and for player B $(0.5,0.5,0)$, which means that player A always wins 4 units of money.

Case2: We transform the crisp game problem to fuzzy game problem and then solve them by three different ranking functions.

Now we consider the payoff matrix as trapezoidal fuzzy numbers in the problem as follows:

Let $\Delta_{1}=0.5$ and $\Delta_{2}=1.5$ for $R_{1}, \Delta_{1}=0.4$ and $\Delta_{2}=1.6$ for $R_{2}, \Delta_{1}=0.25$ and $\Delta_{2}=1.75$ for $R_{3}$

Where $R_{i}=\left(a_{i j}-1, a_{i j}+1, \Delta_{1}, \Delta_{2}\right)$ and $i, j=$ $1,2,3$.

1

Player A

1
2
3 $\left[\begin{array}{ccc}(0,2,0.5,1.5) & (6,8,0.5,1.5) & (1,3,0.5,1.5) \\ (5,7,0.4,1.6) & (1,3,0.4,1.6) & (6,8,0.4,1.6) \\ (4,6,0.25,1.75) & (0,2,0.25,1.75) & (5,7,0.25,1.75)\end{array}\right]$

1- Applying the first ranking algorithm $\mathrm{R}(\tilde{A})$ $=\frac{a+b+c+d}{4}$, then the payoff matrix is as follows:

Player BRow minimum

\begin{tabular}{|c|c|c|c|c|}
\hline \multirow{3}{*}{ Player A } & $1[1$ & 4 & 1.51 & 1 \\
\hline & \begin{tabular}{l|l}
2 & 3.5
\end{tabular} & 1.5 & 4 & 1.5 \\
\hline & $3[3$ & 1 & 3.5 & 1 \\
\hline
\end{tabular}

Column maximum $3.5 \quad 4 \quad 4$

By using principle of dominance to reduce the size of game matrix into $(2 \times 2)$ then using oddments method. The value of the game is $v=2.5$ units of money with an optimal strategy for player A $(0.4$, $0.6,0)$ and for player $\mathrm{B}(0.5,0.5,0)$, which means that player A always wins 2.5 units of money.

2- Applying the second ranking algorithm $\mathrm{R}(\tilde{A})=\frac{2 a+2 b+d-c}{4}$, then the payoff matrix is as follows:
Player B

\begin{tabular}{ll|ccc|c}
1 & 2 & 3 & \multicolumn{3}{c}{ Row minimum } \\
& 1 & 1.25 & 7.25 & 2.25 & 1.25 \\
Player A & 2 & 6.3 & 2.3 & 7.3 & 2.3 \\
& 3 & 5.375 & 1.375 & 6.375 & 1.375 \\
\multicolumn{2}{c}{ Column maximum } & 6.3 & 7.25 & 7.3
\end{tabular}

By using principle of dominance to reduce the size of payoff matrix into $(2 \times 2)$ then solving it with probabilities the value of the game is $\mathrm{v}=4.28$ units of money with an optimal strategy for player A $(0.4$, $0.6,0)$ and for player $B(0.5,0.5,0)$, which means that player A always wins 4.28 units of money.

3-Applying the third ranking algorithm $\mathrm{R}(\tilde{A})=$ $\frac{w}{4}(a+b+c+d), w \in[0,1]$.

We state all the values of the weight $(w)$ neglecting (0) using the principle of dominance to reduce the size of matrices into $(2 \times 2)$ then solving with probabilities to find the values of the game represented in table (1-1) as follows:

Table 1-1. represents the solution of the third algorithm

\begin{tabular}{cc}
\hline Weight & Values of the game \\
\hline 0.1 & 0.25 \\
0.2 & 0.5 \\
0.3 & 0.75 \\
0.4 & 1 \\
0.5 & 1.25 \\
0.6 & 1.5 \\
0.7 & 1.75 \\
0.8 & 2 \\
0.9 & 2.25 \\
1 & 2.5 \\
\hline
\end{tabular}

Where the best weight that gives the best gains is when $w \geq 0.8$, where player A when $w \in[0.8,1]$ with an optimal strategy for player A $(0.4,0.6,0)$ and for player $\mathrm{B}(0.5,0.5,0)$ for all weights.

\section{Conclusion:}

When the games problem is in the crisp case, the value of the game is $\mathrm{v}=4$ units. After transforming the elements (entries) of the payoff matrix to trapezoidal fuzzy case, we found that the values of the game are the same $v=2.5$ units for the first algorithm and the third when $(w=1)$ while the best gain is when the second algorithm is used where the value of the game is $v=4.28$ units.

Then the second algorithm will be helpful to the decision maker when he deals with fuzzy game problems to get the best gains. 


\section{References:}

1. Gupta PK, Hira DS. Operations Research. First Edition, S. Chand \& company LTD, New Delhi, India.

2. Kumar RS, Gnanaprakash K. Solving Fuzzy Game of Order $3 \times 3$ Using Octagonal Fuzzy Numbers. International Journal of Innovative Science Engineering \& Technology, 2015; 3: 43-53.

3. Jawad MA. Fuzzy Games Theory to Determine the Best Strategy for the Mobile Phone Networks M.Sc Thesis, College of Administration and Economics, University of Baghdad (in Arabic) 2012.

4. Aristidou M, Sarangi S. Games in Fuzzy Enviroments. Louisiana State of University, 2005; pp1-24.

5. Gao J. Credibilistic Game with Fuzzy Information. Journal of uncertain systems, 2007; 1(1): 74-80.

6. Medinechkiene M, Zavadska EK, Turskis Z. Dwelling Selection by Applying Fuzzy Game Theory. Archives of Civil and Mechanical Engineering, XI 2010; (3): 682-697.

7. Kumar RS, Kumaraghura S. Solution of Fuzzy Game Problem Using Triangular Fuzzy Number. International Journal of Innovative Science, Engineering \& Technology, 2015; 2: 497-502.

8. Selvakumari K, Lavanya S. An Approach for Solving Fuzzy Game Problem. Indian journal of Science and Technology, 2015; 8(15): 2-6.

9. Zimmermann HJ. Fuzzy Set Theory. Journal of Advanced Review, 2010; 2: 317-332.
10. Beg I, Ashraf S. Fuzzy Relational Calculas. Lahore, The Bulletin of Malaysian Socitey series 2010; 2, 37(1): 1-79.

11. Narayanamoorthy S, Saranya S, Maheswari S. A Method for Solving Fuzzy Transportation Problem (FTP) Using Fuzzy Russell's Method. I. J. Intelligent Systems and Applications, 2013; 71-75.

12. Hashem HA. Solving Fuzzy Linear Programming with Nonsymmetrical Trapezoidal Fuzzy Number. Journal of Applied Sciences Research, 2013; 9(6): 4001-4005.

13. Maleki HR. Ranking Function and their Application to Fuzzy Linear Programming. Far East J. Math. Sci, 2002; 4: 283-301.

14. Muruganandam DrS, Srinivasan MR. A New Algorithm for Solving Fuzzy Transportation Problems with Trapezoidal Fuzzy Numbers. International Journal of Recent Trends in Engineering \& Research, 2016; 2: 428-437.

15. Al-Kanani IH, Adnan FA. Ranking Function Methods for Solving Fuzzy Linear Programming Problems. Mathematical Theory and Modeling, 2014; 4(4):6572.

16. Kirubha G. Prompting Method for Solving Fuzzy Assignment Problem under Fuzzy Enviroment. Imperial Journal of Interdisciplinary Research (IJIR), 2016; 2: 682-688.

17. Murthy PR. Operations Research. Second Edition, New Age International (P) LTD. New Delhi. 2007.

\section{حل مشكلة الالعاب الضبابيه باستخدام الدوال الرتبيه}

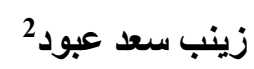

$$
\begin{aligned}
& \text { ايدن حسن حسين } 1 \\
& \text { 1، } 2 \text { قسم الرياضيات، كلية العلوم للبنات، جامعة بغداد، بغداد، العراق. }
\end{aligned}
$$



